\title{
Block-avoiding point sequencings of directed triple systems
}

\author{
Donald L. Kreher ${ }^{1}$, Douglas R. Stinson*2, and Shannon Veitch ${ }^{3}$ \\ ${ }^{1}$ Department of Mathematical Sciences, Michigan Technological \\ University Houghton, MI 49931, U.S.A. \\ ${ }^{2}$ David R. Cheriton School of Computer Science, University of \\ Waterloo, Waterloo, Ontario, N2L 3G1, Canada \\ ${ }^{3}$ Department of Combinatorics and Optimization, University of \\ Waterloo, Waterloo, Ontario, N2L 3G1, Canada
}

July 26, 2019

\begin{abstract}
A directed triple system of order $v$ (or, DTS $(v)$ ) is decomposition of the complete directed graph $\vec{K}_{v}$ into transitive triples. A $v$-good sequencing of a DTS $(v)$ is a permutation of the points of the design, say $\left[\begin{array}{lll}x_{1} & \cdots & x_{v}\end{array}\right]$, such that, for every triple $(x, y, z)$ in the design, it is not the case that $x=x_{i}, y=x_{j}$ and $z=x_{k}$ with $i<j<k$. We prove that there exists a DTS $(v)$ having a $v$-good sequencing for all positive integers $v \equiv 0,1 \bmod 3$. Further, for all positive integers $v \equiv 0,1 \bmod 3, v \geq 7$, we prove that there is a $\operatorname{DTS}(v)$ that does not have a $v$-good sequencing. We also derive some computational results concerning $v$-good sequencings of all the nonisomorphic DTS $(v)$ for $v \leq 7$.
\end{abstract}

\section{Introduction}

A Steiner triple system of order $v$ is a pair $(X, \mathcal{B})$, where $X$ is a set of $v$ points and $\mathcal{B}$ is a set of 3 -subsets of $X$ (called blocks), such that every pair of points occurs in exactly one block. We will abbreviate the phrase "Steiner

*D.R. Stinson's research is supported by NSERC discovery grant RGPIN-03882. 
triple system of order $v$ " to STS $(v)$. It is well-known that an $\operatorname{STS}(v)$ contains exactly $v(v-1) / 6$ blocks, and an $\operatorname{STS}(v)$ exists if and only if $v \equiv 1,3 \bmod 6$. The definitive reference for Steiner triple systems is the book [5] by Colbourn and Rosa.

There are two directed variants of STS $(v)$, which are known as Mendelsohn triple systems and directed triple systems. We study directed triple systems in this paper. First, we define a transitive triple to be an ordered triple $(x, y, z)$, where $x, y, z$ are distinct. This triple contains the directed edges $(x, y),(x, z)$ and $(y, z)$ (we might also write these directed edges as $x y, x z$ and $y z$, respectively). Thus, the triple $(x, y, z)$ can be thought of as the following directed graph:

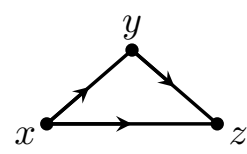

Let $X$ be a set of $v$ points or vertices and let $\vec{K}_{v}$ denote the complete directed graph on vertex set $X$. This graph has $v(v-1)$ directed edges. A directed triple system of order $v$ is a pair $(X, \mathcal{B})$, where $X$ is a set of $v$ points and $\mathcal{B}$ is a set of transitive triples (or more simply, triples) whose elements are members of $X$, such that every directed edge in $\vec{K}_{v}$ occurs in exactly one triple in $\mathcal{B}$. (Thus, the triples in a directed triple system fulfill the same role as blocks in a Steiner triple system.)

We will abbreviate the phrase "directed triple system of order $v$ " to $\operatorname{DTS}(v)$. It is well-known that a DTS $(v)$ contains exactly $v(v-1) / 3$ triples, and a $\operatorname{DTS}(v)$ exists if and only if $v \equiv 0,1 \bmod 3$. Various results on DTS $(v)$ can be found in [5].

The following problem on sequencing points in an STS $(v)$ was introduced by Kreher and Stinson in [9] and studied further in Stinson and Veitch [13]. Suppose $(X, \mathcal{B})$ is an $\operatorname{STS}(v)$ and let $\ell \geq 3$ be an integer. A sequencing of the $\operatorname{STS}(v)$ is a permutation $\pi=\left[\begin{array}{llll}x_{1} & x_{2} & \cdots & x_{v}\end{array}\right]$ of $X$. A sequencing $\pi=\left[\begin{array}{llll}x_{1} & x_{2} & \cdots & x_{v}\end{array}\right]$ is $\ell$-good if no $\ell$ consecutive points in $\pi$ contain a block in $\mathcal{B}$.

Some related but different sequencing problems for STS $(v)$ are studied in [2] and [8]. Also, for a recent survey paper on this topic, see [1].

It is obvious that an STS $(v)$ cannot have a $v$-good sequencing. In fact, it was shown in [13] that, if an $\operatorname{STS}(v)$ with $v \geq 7$ has an $\ell$-good sequencing, then $\ell<(v+2) / 3$.

In this paper, we study the corresponding question for DTS $(v)$. Let $(X, \mathcal{B})$ be a $\operatorname{DTS}(v)$. We first need to define what it means for a sequencing 
$\pi$ of $(X, \mathcal{B})$ to "contain" a particular transitive triple. The most natural approach seems to be to regard the sequencing as a total ordering defined on the points in $X$. A triple $(x, y, z) \in \mathcal{B}$ is said to be contained in $\ell$ consecutive points of the sequencing $\pi=\left[x_{1} x_{2} \cdots x_{v}\right]$ if

1. $\{x, y, z\} \subseteq\left\{x_{i}, x_{i+1}, \ldots, x_{i+\ell-1}\right\}$ for some $i$, and

2. $x<y<z$ in the sequencing.

Then, a sequencing $\pi$ is $\ell$-good if no $\ell$ consecutive points in the sequencing contain a triple in $\mathcal{B}$.

Unlike STS $(v)$, it is possible that a DTS $(v)$ has a $v$-good sequencing. Informally, this just means that for every triple $(x, y, x)$ in the DTS $(v)$, the ordering of $x, y$ and $z$ in the sequencing is not $x<y<z$.

Example 1.1. Let $X=\{1,2,3\}$ and $\mathcal{B}=\{(1,2,3),(3,2,1)\}$. Then $(X, \mathcal{B})$

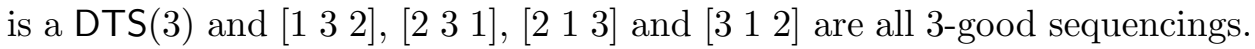

Example 1.2. Let $X=\{1,2,3,4\}$ and $\mathcal{B}=\{(1,2,3),(2,1,4),(3,4,2),(4,3,1)\}$. Then $(X, \mathcal{B})$ is a DTS $(4)$ and $\left[\begin{array}{llll}1 & 3 & 2 & 4\end{array}\right]$ is a 4 -good sequencing.

Example 1.3. Let $X=\{\infty\} \cup \mathbb{Z}_{5}$ and $\mathcal{B}=\{(0, \infty, 4),(0,1,3)\} \bmod 5$. Then $(X, \mathcal{B})$ is a $\operatorname{DTS}(6)$ and $\left[\begin{array}{lllll}\infty & 0 & 4 & 4 & 1\end{array}\right]$ is a 6 -good sequencing.

\section{$1.1 \quad$ Summary of results}

In Section 2, we use recursive constructions to prove that there exists a $\operatorname{DTS}(v)$ having a $v$-good sequencing for all positive integers $v \equiv 0,1 \mathrm{mod}$ 3. In Section 3, we report some computational results concerning $v$-good sequencings of all the nonisomorphic DTS $(v)$ for $v \leq 7$. Perhaps surprisingly, there are precisely four nonisomorphic DTS(7) (out of a total of 2368) that do not have 7-good sequencings. In Section 4, we investigate a possible algorithmic approach to prove that a given DTS $(v)$ does not have a $v$-good sequencing. We illustrate by providing a short proof that a certain DTS(7) does not have a 7 -good sequencing. We also use the same technique to enable the construction of $\operatorname{DTS}(v)$ that do not have $v$-good sequencings for $v=$ $9,10,12,13,16$ and 18. Then, in Section 5, we use recursive constructions to prove that there is a DTS $(v)$ that does not have a $v$-good sequencing for all positive integers $v \equiv 0,1 \bmod 3, v \geq 7$. 


\section{Constructions}

We provide two proofs that there exists a DTS $(v)$ having a $v$-good sequencing for all $v \equiv 0,1 \bmod 3$. First, we give a PBD proof. Then we present a proof using two well-known recursive "doubling" constructions for DTS, having the form $v \rightarrow 2 v+1$ and $v \rightarrow 2 v+4$.

\section{$2.1 \quad$ PBD-closure}

Let $K$ be a set whose elements are all integers $\geq 2$. A pair $(X, \mathcal{B})$ is a $(v, K)$-pairwise balanced design (or, $(v, K)-P B D)$ if $X$ is a set of $v$ points and $\mathcal{B}$ is a set of subsets of $X$ (called blocks) such that

- every pair of points from $X$ occurs in exactly one block in $\mathcal{B}$, and

- $|B| \in K$ for every $B \in \mathcal{B}$.

A set $K$, whose elements are all integers $\geq 2$, is $P B D$-closed if $v \in K$ whenever there exists a $(v, K)$-PBD.

Let $K_{\mathrm{DTS}}=\{v \geq 3$ : there exists a DTS $(v)$ having a $v$-good sequencing $\}$. We show that $K_{\mathrm{DTS}}$ is PBD-closed.

Theorem 2.1. $K_{\mathrm{DTS}}$ is PBD-closed.

Proof. Suppose $(X, \mathcal{B})$ is a $\left(v, K_{\mathrm{DTS}}\right)$-PBD, where $X=\{1, \ldots, v\}$. We will construct a DTS $(v)$ having a $\left[\begin{array}{llll}1 & 2 & \cdots & v\end{array}\right]$ as a $v$-good sequencing.

Let $B \in \mathcal{B}$, say $B=\left\{x_{1}, x_{2}, \ldots, x_{k}\right\}$, where $x_{1}<\cdots<x_{k}$. There is $\operatorname{DTS}(k)$ having a $k$-good sequencing. Therefore, by relabelling points, there exists a $\operatorname{DTS}(k)$, say $\left(B, \mathcal{A}_{B}\right)$, for which $\left[\begin{array}{llll}x_{1} & x_{2} & \cdots & x_{k}\end{array}\right]$ is a $k$-good sequencing.

Define

$$
\mathcal{A}=\bigcup_{B \in \mathcal{B}} \mathcal{A}_{B}
$$

It is straightforward to verify that $(X, \mathcal{A})$ is a $\operatorname{DTS}(v)$ for which $\left[\begin{array}{llll}1 & 2 & \cdots & v\end{array}\right]$ is a $v$-good sequencing.

Corollary 2.2. There exists a DTS $(v)$ having a v-good sequencing if and only if $v \equiv 0,1 \bmod 3$.

Proof. We have already noted that $v \equiv 0,1 \bmod 3$ is a necessary condition for existence of a $\operatorname{DTS}(v)$.

We prove sufficiency as follows. For $v \equiv 0,1 \bmod 3, v \geq 7$, there exists a $(v,\{3,4\})$-PBD (see [4, Table IV.3.23]). We know that $3,4 \in K_{\text {DTs }}$ from 
Examples 1.1 and 1.2. Then we can apply Theorem 2.1 to show that $v \in$ $K_{\text {DTS }}$. Finally, $6 \in K_{\text {DTS }}$ from Example 1.3 .

\subsection{Doubling constructions}

In this section, we prove the existence of a $v$-good sequencing of a DTS $(v)$ by using two doubling constructions. The two constructions we use can be found in [5, §24, Lemma 1.1 and Lemma 1.2], for example.

Lemma 2.3. If there exists a DTS $(v)$ having a v-good sequencing, then there exists a DTS $(2 v+1)$ having a $(2 v+1)$-good sequencing.

Proof. Let $(X=\{1, \ldots, v\}, \mathcal{B})$ be a $\operatorname{DTS}(v)$ having a $v$-good sequencing $\left[\begin{array}{llll}1 & 2 & \cdots & v\end{array}\right]$. Let $L$ be a latin square of order $v+1$ having constant diagonal, whose rows and columns are indexed by the set $Y=\{v+1, \ldots, 2 v+1\}$ of size $v+1$ and whose off-diagonal symbols are from $X$. Form a set $\mathcal{C}$ of triples as follows: For each $i, j \in Y, i \neq j$, let $(i, L(i, j), j) \in \mathcal{C}$. Then, $(X \cup Y, \mathcal{B} \cup \mathcal{C})$ is a $\operatorname{DTS}(2 v+1)$.

It is not hard to see that $(2 v+1)$-good sequencing of this DTS is given by

$$
[12 \cdots v v+1 \cdots 2 v+1]
$$

This follows, because

1. $\left[\begin{array}{llll}1 & 2 & \cdots & v\end{array}\right]$ is a $v$-good sequencing of the triples in $\mathcal{B}$, and

2. for each triple $(i, L(i, j), j) \in \mathcal{C}$, the point $L(i, j)$ occurs in the sequencing before the point $i$.

Lemma 2.4. If there exists a DTS $(v)$ having a v-good sequencing, then there exists a DTS $(2 v+4)$ having a $(2 v+4)$-good sequencing.

Proof. Let $\left(X=\left\{x_{1}, \ldots, x_{v}\right\}, \mathcal{B}\right)$ be a DTS $(v)$ having the $v$-good sequencing $\left[\begin{array}{llll}x_{1} & x_{2} & \cdots & x_{v}\end{array}\right]$. Let $Y=\mathbb{Z}_{v+4}$ be disjoint from $X$. Form $v$ disjoint sets $S_{1}, \ldots, S_{v}$, each consisting of $v+4$ ordered pairs of points from $Y$, by taking

$$
S_{i}=\{(a, b): b-a \equiv i \bmod (v+4)\}
$$

for $i=1, \ldots, v$. Now form a set $\mathcal{C}$ comprised of the following triples:

1. for each $i, 1 \leq i \leq v$, and for every $(a, b) \in S_{i}$, the triple $\left(a, x_{i}, b\right) \in \mathcal{C}$, and 
2. for each $i \in \mathbb{Z}_{v+4}$, the triple $(i, v+2+i, v+1+i) \bmod (v+4) \in \mathcal{C}$.

Then $(X \cup Y, \mathcal{B} \cup \mathcal{C})$ is a $\operatorname{DTS}(2 v+4)$.

We claim that a $(2 v+4)$-good sequencing of this DTS is given by

$$
\left[x_{1} x_{2} \cdots x_{v} \quad \begin{array}{lllll} 
& \cdots & \cdots & v & +3
\end{array}\right] .
$$

The first $v$ points do not contain a triple because $\left[\begin{array}{llll}x_{1} & x_{2} & \cdots & x_{v}\end{array}\right]$ is a $v$ good sequencing of the triples in $\mathcal{B}$. For each of the triples $\left(a, x_{i}, b\right) \in \mathcal{C}$ constructed in 1., $x_{i}$ occurs in the sequencing before the point $a$. Also, for each of the triples in $(i, v+2+i, v+1+i) \in \mathcal{C}$ constructed in 2 , either

1. $v+2+i \bmod (v+4)>v+1+i \bmod (v+4)$, or

2. $v+2+i=0$, in which case $i>0=v+2+i$.

So, the sequencing is $(2 v+4)$-good.

These lemmas suffice to prove the desired existence result.

Theorem 2.5. There exists a DTS $(v)$ having a v-good sequencing if and only if $v \equiv 0,1 \bmod 3$.

Proof. We have already noted that $v \equiv 0,1 \bmod 3$ is a necessary condition for existence of a DTS $(v)$, and there exists a DTS $(v)$ with a $v$-good sequencing for $v=3,4$, and 6 . We proceed by induction. Suppose $v>6$, $v \equiv 0,1 \bmod 3$. If $v$ is odd, write $v=2 k+1$. Then $k \equiv 0,1 \bmod 3$, so by induction, it follows that there exists a $\operatorname{DTS}(k)$ having a $k$-good sequencing. Hence, there exists a DTS $(v)$ having a $v$-good sequencing by applying Lemma 2.3. Similarly, if $v$ is even, write $v=2 k+4$ and apply Lemma 2.4.

\section{Computational results}

In this section, we report our results on $v$-good sequencings of DTS $(v)$, for $v \leq 7$. The nonisomorphic $\operatorname{DTS}(v)$ for $v \leq 7$ have been enumerated by Colbourn and Colbourn [3] (see also [11]). We can test a DTS $(v)$ by exhaustively checking all $v$ ! permutations to see which of them are $v$-good sequencings. This does not take very much time for these small values of $v$.

Up to isomorphism, there is a unique DTS(3) and it has a 3-good sequencing, as shown in Example 1.1.

There are three nonisomorphic DTS(4). We present the three designs, along with 4-good sequencings: 
$\mathcal{D}_{4} \mathbf{1}: \quad(0,3,2) \quad(1,2,3) \quad(2,1,0) \quad(3,0,1)$

4-good sequencing: 0213

number of 4-good sequencings: 8

$\mathcal{D}_{4} \mathbf{2}: \quad(0,3,2) \quad(1,2,3) \quad(2,0,1) \quad(3,1,0)$

4-good sequencing: 0213

number of 4-good sequencings: 8

$\mathcal{D}_{4} 3: \quad(0,3,2) \quad(1,2,0) \quad(2,1,3) \quad(3,0,1)$

4-good sequencing: 0123

number of 4-good sequencings: 8

There are 32 nonisomorphic DTS(6) and they all have 6-good sequencings. The designs and their 6 -good sequencings are presented in the technical report [10].

There are exactly 2368 nonisomorphic DTS(7). We construct these following the method described in [11. There are four nonisomorphic $(v, 3,2)$ BIBDs (or TTS $(7)$ ), which we denote $\mathcal{D}_{7} 1, \mathcal{D}_{7} 2, \mathcal{D}_{7} 3$ and $\mathcal{D}_{7} 4$. The triples in these four designs are directed in all possible ways to form DTS(7) and then isomorphic designs are eliminated. It turns out that all but four of the nonisomorphic DTS(7) have 7-good sequencings. These 7 -good sequencings are all presented in [10].

The results can be summarized as follows:

- 18 DTS(7) have $\mathcal{D}_{7} 1$ as the underlying TTS(7). All of these DTS(7) have 7 -good sequencings.

- 274 DTS(7) have $\mathcal{D}_{7} 2$ as the underlying TTS(7). All of these DTS(7) have 7 -good sequencings.

- 1060 DTS(7) have $\mathcal{D}_{7} 3$ as the underlying TTS(7). All of these DTS(7) have 7 -good sequencings.

- 1016 DTS(7) have $\mathcal{D}_{7} 4$ as the underlying TTS(7). 1012 of these DTS(7) have 7 -good sequencings.

It is interesting to note that the four DTS(7) that do not have 7-good sequencings all have a 6 -good sequencing. These four DTS(7), along with 6 -good sequencings, are as follows: 


$$
\begin{array}{llllllll}
\mathcal{D}_{7} 4.926 & (0,4,2) & (0,5,6) & (1,3,0) & (1,5,2) & (2,0,1) & (2,6,5) & (3,1,6) \\
& (3,2,4) & (4,3,5) & (4,6,1) & (5,0,3) & (5,1,4) & (6,2,3) & (6,4,0)
\end{array}
$$

6-good sequencing: 0123456

number of 6-good sequencings: 124

$$
\begin{array}{llllllll}
\mathcal{D}_{7} 4.958: & (0,4,2) & (0,5,3) & (1,5,2) & (1,6,3) & (2,1,0) & (2,3,4) & (3,0,1) \\
(3,2,6) & (4,3,5) & (4,6,0) & (5,0,6) & (5,1,4) & (6,2,5) & (6,4,1)
\end{array}
$$

6-good sequencing: 0245613

number of 6-good sequencings: 124

$\begin{array}{llllllll}\mathcal{D}_{7} 4.1015: & (0,3,5) & (0,4,2) & (1,2,5) & (1,6,3) & (2,1,0) & (2,3,6) & (3,0,1) \\ & (3,2,4) & (4,0,6) & (4,5,3) & (5,4,1) & (5,6,0) & (6,1,4) & (6,5,2)\end{array}$

6-good sequencing: 0153462

number of 6-good sequencings: 112

$\begin{array}{llllllll}\mathcal{D}_{7} 4.1016:: & (0,3,1) & (0,4,6) & (1,2,0) & (1,6,4) & (2,1,5) & (2,3,4) & (3,0,5) \\ & (3,2,6) & (4,0,2) & (4,5,1) & (5,4,3) & (5,6,2) & (6,1,3) & (6,5,0)\end{array}$

6-good sequencing: 0124356

number of 6-good sequencings: 112

It does not seem feasible to test all the DTS(9) because it is shown in [1] that there are 596, 893, 386 nonisomorphic DTS(9).

\section{Algorithmic approaches}

It is of interest to devise an algorithm to determine if a given DTS $(v)$ can be sequenced. Obviously, checking all $v$ ! permutations is not practical for large values of $v$, so we would like to have a more efficient algorithm.

Here is one possible approach that could be considered. A directed triple $(a, b, c)$ in a DTS $(v)$ leads to the following necessary condition for the existence of a $v$-good sequencing of $v$ points:

$$
(c<b) \vee(b<a) .
$$

For each of the $v(v-1) / 3$ triples in a DTS $(v)$, we obtain a condition similar to (11). Suppose, for each of the triples, we choose one of the two relevant inequalities (i.e., for the triple $(a, b, c)$, we choose $c<b$, or we choose $b<a$ ). We can interpret an inequality as an edge in a directed graph, i.e., $c<b$ corresponds to the directed edge $(c, b)$ and $b<a$ corresponds to the directed 
edge $(b, a)$. Thus we obtain by this method a directed graph $\mathcal{D}$, on the $v$ points of the $\operatorname{DTS}(v)$, having $e=v(v-1) / 3$ edges.

It is easy to determine in polynomial time if this graph $\mathcal{D}$ has a topological ordering (i.e., a total ordering of the points that is compatible with all the edges in the directed graph). A topological ordering is clearly a $v$-good sequencing of the given DTS $(v)$. It is well-known that there is a topological ordering of a directed graph if and only if the graph is a DAG (directed acyclic graph). Further, testing a directed graph to see if it has a topological ordering can be done using a simple modification of DFS (depth-first search). The complexity of this algorithm (given a particular graph $\mathcal{D}$ ) is $O(v+e)=$ $O\left(v^{2}\right)$. (For these results, see, for example, [6, §22].)

We could construct all the possible directed graphs and test each of them in this way. If none of the graphs are DAGs, then the DTS $(v)$ does not have a $v$-good sequencing. The problem is that there are $2^{v(v-1) / 3}$ graphs to test, so this is not a polynomial-time algorithm. However, in practice, we can often achieve a significant reduction in the number of graphs to be considered. It is possible that this approach might lead to a fairly simple proof that a given DTS $(v)$ has no $v$-good sequencing. This technique works well in practice for small values of $v$ and it can even be done by hand with a bit of patience. We illustrate by deriving a proof that the DTS(7) named $\mathcal{D}_{7} 4.926$ (which was presented in Section 3) has no 7-good sequencing.

Theorem 4.1. The DTS(7) named $\mathcal{D}_{7} 4.926$ does not have a 7 -good sequencing.

Proof. First, we list the triples in $\mathcal{D}_{7} 4.926$, along with the conditions derived from them, in Table 1. The idea is to show that any directed graph that satisfies the required conditions for every triple must contain a directed cycle. We begin by considering triple $T_{1}$. The proof divides into two cases:

Case 1: $4<0$

Case $2: 2<4$ 
Table 1: The triples in $D(7) 4.926$

\begin{tabular}{c|c} 
triple & condition \\
\hline$T_{1}=(0,4,2)$ & $(4<0) \vee(2<4)$ \\
$T_{2}=(0,5,6)$ & $(5<0) \vee(6<5)$ \\
$T_{3}=(1,3,0)$ & $(3<1) \vee(0<3)$ \\
$T_{4}=(1,5,2)$ & $(5<1) \vee(2<5)$ \\
$T_{5}=(2,0,1)$ & $(0<2) \vee(1<0)$ \\
$T_{6}=(2,6,5)$ & $(6<2) \vee(5<6)$ \\
$T_{7}=(3,1,6)$ & $(1<3) \vee(6<1)$ \\
$T_{8}=(3,2,4)$ & $(2<3) \vee(4<2)$ \\
$T_{9}=(4,3,5)$ & $(3<4) \vee(5<3)$ \\
$T_{10}=(4,6,1)$ & $(6<4) \vee(1<6)$ \\
$T_{11}=(5,0,3)$ & $(0<5) \vee(3<0)$ \\
$T_{12}=(5,1,4)$ & $(1<5) \vee(4<1)$ \\
$T_{13}=(6,2,3)$ & $(2<6) \vee(3<2)$ \\
$T_{14}=(6,4,0)$ & $(4<6) \vee(0<4)$
\end{tabular}

For case 1 , we assume $4<0$ and we proceed as follows:

$$
\begin{aligned}
& T_{14} \quad \Longrightarrow \quad(4<6) \vee(0<4) \text {, so } 4<6 \\
& T_{10} \quad \Longrightarrow \quad(6<4) \vee(1<6) \text {, so } 1<6 \\
& T_{7} \Longrightarrow(1<3) \vee(6<1) \text {, so } 1<3 \\
& T_{3} \Longrightarrow(3<1) \vee(0<3) \text {, so } 0<3 \\
& T_{11} \quad \Longrightarrow \quad(0<5) \vee(3<0) \text {, so } 0<5 \\
& T_{2} \quad \Longrightarrow \quad(5<0) \vee(6<5) \text {, so } 6<5 \\
& T_{6} \quad \Longrightarrow \quad(6<2) \vee(5<6) \text {, so } 6<2 \\
& T_{13} \quad \Longrightarrow \quad(2<6) \vee(3<2) \text {, so } 3<2 \\
& T_{8} \quad \Longrightarrow \quad(2<3) \vee(4<2) \text {, so } 4<2 \text {. }
\end{aligned}
$$

So far, there are no directed cycles, so we proceed a bit further.

$$
T_{4} \quad \Longrightarrow \quad(5<1) \vee(2<5) \text {. }
$$

If $5<1$, then $1<6<5<1$ is a directed cycle. Therefore $2<5$.

$$
T_{9} \quad \Longrightarrow \quad(3<4) \vee(5<3) \text {. }
$$


If $3<4$, then we get the directed cycle $3<4<0<3$. Therefore $5<3$. But this creates the directed cycle $5<3<2<5$. Thus Case 1 is impossible.

Now we turn to Case 2 , where we assume $2<4$. We proceed as follows:

$$
\begin{aligned}
& T_{8} \quad \Longrightarrow \quad(2<3) \vee(4<2) \text {, so } 2<3 \\
& T_{13} \quad \Longrightarrow \quad(2<6) \vee(3<2) \text {, so } 2<6 \\
& T_{6} \quad \Longrightarrow \quad(6<2) \vee(5<6) \text {, so } 5<6 \\
& T_{2} \quad \Longrightarrow \quad(5<0) \vee(6<5) \text {, so } 5<0 \\
& T_{11} \quad \Longrightarrow \quad(0<5) \vee(3<0) \text {, so } 3<0 \\
& T_{3} \quad \Longrightarrow \quad(3<1) \vee(0<3) \text {, so } 3<1 \\
& T_{7} \quad \Longrightarrow \quad(1<3) \vee(6<1) \text {, so } 6<1 \\
& T_{10} \quad \Longrightarrow \quad(6<4) \vee(1<6) \text {, so } 6<4 \\
& T_{14} \quad \Longrightarrow \quad(4<6) \vee(0<4) \text {, so } 0<4 \text {. }
\end{aligned}
$$

We continue.

$$
T_{5} \quad \Longrightarrow \quad(0<2) \vee(1<0) \text {. }
$$

If $0<2$, then $3<0<2<3$ is a directed cycle. Therefore $1<0$.

$$
T_{12} \Longrightarrow(1<5) \vee(4<1) .
$$

If $1<5$, then $6<1<5<6$ is a directed cycle. Therefore $4<1$ But then $0<4<1<0$ is a directed cycle. Thus Case 2 is also impossible.

Similar reasoning can be used to show that the DTS(7) named $\mathcal{D}_{7} 4.958$, $\mathcal{D}_{7} 4.1015$, and $\mathcal{D}_{7} 4.1016$ (all of which are presented in Section 3) have no 7 -good sequencings.

We are also able to use this technique to construct DTS $(v)$ that can be proven not to have a $v$-good sequencing for $v \in\{9,10,12,13,16,18\}$. Our proof depends on the following lemma.

Lemma 4.2. Suppose that a DTS $(v)$ contains the following twelve triples:

$$
\begin{array}{llllll}
(1,2,3) & (4,3,2) & (3,4,5) & (6,5,4) & (5,6,2) & (7,2,6) \\
(2,7,8) & (3,8,7) & (8,3,6) & (2,1,0) & (6,0,1) & (0,6,3)
\end{array}
$$

Then the DTS $(v)$ cannot have a v-good sequencing. 
Table 2: Twelve triples

\begin{tabular}{c|c} 
triple & condition \\
\hline$T_{1}=(1,2,3)$ & $(2<1) \vee(3<2)$ \\
$T_{2}=(4,3,2)$ & $(3<4) \vee(2<3)$ \\
$T_{3}=(3,4,5)$ & $(4<3) \vee(5<4)$ \\
$T_{4}=(6,5,4)$ & $(5<6) \vee(4<5)$ \\
$T_{5}=(5,6,2)$ & $(6<5) \vee(2<6)$ \\
$T_{6}=(7,2,6)$ & $(2<7) \vee(6<2)$ \\
$T_{7}=(2,7,8)$ & $(7<2) \vee(8<7)$ \\
$T_{8}=(3,8,7)$ & $(8<3) \vee(7<8)$ \\
$T_{9}=(8,3,6)$ & $(3<8) \vee(6<3)$ \\
$T_{10}=(2,1,0)$ & $(1<2) \vee(0<1)$ \\
$T_{11}=(6,0,1)$ & $(0<6) \vee(1<0)$ \\
$T_{12}=(0,6,3)$ & $(6<0) \vee(3<6)$
\end{tabular}

Proof. First, we list the twelve triples, along with the conditions derived from them, in Table 2. We begin by considering triple $T_{1}$. The proof divides into two cases:

Case $1: 3<2$

Case $2: 2<1$

For Case 1 , we assume $3<2$. We proceed as follows:

$$
\begin{aligned}
& T_{2} \Longrightarrow(3<4) \vee(2<3), \text { so } 3<4 \\
& T_{3} \Longrightarrow(4<3) \vee(5<4), \text { so } 5<4 \\
& T_{4} \Longrightarrow(5<6) \vee(4<5), \text { so } 5<6 \\
& T_{5} \Longrightarrow(6<5) \vee(2<6), \text { so } 2<6 \\
& T_{6} \Longrightarrow(2<7) \vee(6<2), \text { so } 2<7 \\
& T_{7} \Longrightarrow(7<2) \vee(8<7), \text { so } 8<7 \\
& T_{8} \Longrightarrow(8<3) \vee(7<8), \text { so } 8<3 \\
& T_{9} \Longrightarrow(3<8) \vee(6<3), \text { so } 6<3 .
\end{aligned}
$$

But then $3<2<6<3$ is a directed cycle. Thus Case 1 is impossible. 
Now we consider Case 2 , where we assume $2<1$. Then we proceed as follows:

$$
\begin{array}{r}
T_{10} \Longrightarrow(1<2) \vee(0<1), \text { so } 0<1 \\
T_{11} \Longrightarrow(0<6) \vee(1<0), \text { so } 0<6 \\
T_{12} \Longrightarrow(6<0) \vee(3<6), \text { so } 3<6 \\
T_{9} \Longrightarrow(3<8) \vee(6<3), \text { so } 3<8 \\
T_{8} \Longrightarrow(8<3) \vee(7<8), \text { so } 7<8 \\
T_{7} \Longrightarrow(7<2) \vee(8<7), \text { so } 7<2 \\
T_{6} \Longrightarrow(2<7) \vee(6<2), \text { so } 6<2 \\
T_{5} \Longrightarrow(6<5) \vee(2<6), \text { so } 6<5 \\
T_{4} \Longrightarrow(5<6) \vee(4<5), \text { so } 4<5 \\
T_{3} \Longrightarrow(4<3) \vee(5<4), \text { so } 4<3 \\
T_{2} \Longrightarrow(3<4) \vee(2<3), \text { so } 2<3 .
\end{array}
$$

But then $3<6<2<3$ is a directed cycle. Thus Case 2 is also impossible.

For $v \in\{9,10,12,13,16,18\}$, we have constructed DTS $(v)$ that contain the twelve triples listed in Lemma 4.2, see Examples 4.14.6. The construction of these DTS $(v)$ made use of a hill-climbing algorithm that is similar to the hill-climbing algorithm to construct STS $(v)$ that is presented in [12].

We provide a brief description of the hill-climbing approach we used. The algorithm attempts to construct a DTS $(v)$ by using three heuristics, which we name $H_{1}, H_{2}$ and $H_{3}$. In the following, $x, y$ and $z$ refer to points in the $\operatorname{DTS}(v)$ that we are constructing.

$\boldsymbol{H}_{1}$ If there exists a point $x$ such that there are at least two points $y, z$ such that the directed edges $x y$ and $x z$ have not occurred in a triple, then construct the triple $(x, y, z)$ and add it to the design. If the directed edge $y z$ already appears in a triple, then delete that triple.

$\boldsymbol{H}_{\mathbf{2}}$ If there exists a point $x$ such that there are two points $y, z$ such that the directed edges $y x$ and $x z$ have not occurred in a triple, then construct the triple $(y, x, z)$ and add it to the design. If the directed edge $y z$ already appears in a triple, then delete that triple. 
$\boldsymbol{H}_{3}$ If there exists a point $x$ such that there are at least two points
$y, z$ such that the directed edges $y x$ and $z x$ have not occurred in a triple,
then construct the triple $(y, z, x)$ and add it to the design. If $y z$ already
appears in a triple then delete that triple.

The hill-climbing algorithm would randomly apply $H_{1}, H_{2}$ and $H_{3}$ over and over again, until (hopefully) a design is constructed. However, we are trying to do something a bit more complicated, namely, to construct a DTS $(v)$ that contains the twelve initial triples listed in Lemma 4.2. Thus, we begin with the initial triples and we need to modify $H_{1}, H_{2}$ and $H_{3}$ so that we never delete an initial triple. This is straightforward; for example, $H_{1}$ would be replaced by the following modified heuristic.

$\boldsymbol{H}_{1}^{*} \quad$ Suppose there exists a point $x$ such that there are at least two points $y, z$ such that the directed edges $x y$ and $x z$ have not occurred in a triple.

1. If there is no existing triple containing the directed edge $y z$, then construct the triple $(x, y, z)$ and add it to the design.

2. If there is a non-initial triple containing the directed edge $y z$, then delete that triple and add the triple $(x, y, z)$ to the design.

3. If there is an initial triple containing the directed edge $y z$, then do nothing (the heuristic fails in this case).

$H_{2}$ and $H_{3}$ would be modified in a similar fashion as $H_{1}$.

As we mentioned above, we used this hill-climbing algorithm to find several DTS $(v)$ that do not have $v$-good sequencings. It should be emphasized that the algorithm is very fast and it ran almost instantaneously on a laptop for the small designs we considered.

Example 4.1. A DTS(9) that has no 9-good sequencing.

$$
\begin{array}{llllllll}
(1,2,3) & (4,3,2) & (3,4,5) & (6,5,4) & (5,6,2) & (7,2,6) & (2,7,8) & (3,8,7) \\
(8,3,6) & (2,1,0) & (6,0,1) & (0,6,3) & (1,7,5) & (5,3,1) & (0,2,4) & (5,0,7) \\
(8,2,5) & (1,6,8) & (7,3,0) & (0,5,8) & (8,4,0) & (4,6,7) & (4,8,1) & (7,1,4)
\end{array}
$$

Example 4.2. A DTS(10) that has no 10-good sequencing.

$$
\begin{array}{llllllll}
(1,2,3) & (4,3,2) & (3,4,5) & (6,5,4) & (5,6,2) & (7,2,6) & (2,7,8) & (3,8,7) \\
(8,3,6) & (2,1,0) & (6,0,1) & (0,6,3) & (0,9,7) & (7,3,1) & (0,2,4) & (1,8,4) \\
(1,5,7) & (9,2,5) & (4,1,9) & (7,9,4) & (9,3,0) & (5,0,8) & (6,9,8) & (9,1,6) \\
(8,2,9) & (5,3,9) & (8,5,1) & (4,8,0) & (4,6,7) & (7,0,5) & &
\end{array}
$$


Example 4.3. A DTS(12) that has no 12-good sequencing.

$$
\begin{array}{lllllll}
(1,2,3) & (4,3,2) & (3,4,5) & (6,5,4) & (5,6,2) & (7,2,6) & (2,7,8) \\
(3,8,7) & (8,3,6) & (2,1,0) & (6,0,1) & (0,6,3) & (11,4,7) & (11,5,3) \\
(6,7,9) & (11,0,2) & (4,1,8) & (5,0,7) & (11,1,6) & (6,11,10) & (5,11,8) \\
(9,4,6) & (7,4,10) & (10,4,0) & (0,9,5) & (9,0,8) & (10,7,5) & (2,10,11) \\
(7,3,0) & (3,11,9) & (9,7,1) & (10,3,1) & (8,1,4) & (8,0,10) & (8,2,5) \\
(10,9,2) & (10,6,8) & (9,3,10) & (5,1,10) & (0,4,11) & (2,4,9) & (1,5,9) \\
(8,9,11) & (1,7,11) & & & & &
\end{array}
$$

Example 4.4. A DTS(13) that has no 13-good sequencing.

$$
\begin{array}{lllllll}
(1,2,3) & (4,3,2) & (3,4,5) & (6,5,4) & (5,6,2) & (7,2,6) & (2,7,8) \\
(3,8,7) & (8,3,6) & (2,1,0) & (6,0,1) & (0,6,3) & (10,4,6) & (7,1,4) \\
(0,11,8) & (6,11,12) & (9,5,10) & (10,8,2) & (12,1,11) & (1,10,5) & (4,12,8) \\
(7,3,11) & (10,11,3) & (8,12,5) & (11,9,6) & (6,8,10) & (0,12,2) & (5,7,0) \\
(4,10,0) & (2,9,12) & (6,7,9) & (7,5,12) & (9,11,7) & (10,1,7) & (9,2,4) \\
(11,4,1) & (12,4,7) & (1,12,6) & (9,1,8) & (5,9,3) & (12,10,9) & (5,8,1) \\
(0,4,9) & (8,9,0) & (3,1,9) & (0,7,10) & (3,10,12) & (8,4,11) & (12,3,0) \\
(11,0,5) & (2,5,11) & (11,2,10) & & & &
\end{array}
$$

Example 4.5. A DTS(16) that has no 16-good sequencing.

$$
\begin{array}{lllllll}
(1,2,3) & (4,3,2) & (3,4,5) & (6,5,4) & (5,6,2) & (7,2,6) & (2,7,8) \\
(3,8,7) & (8,3,6) & (2,1,0) & (6,0,1) & (0,6,3) & (8,10,13) & (4,14,1) \\
(7,0,9) & (14,10,12) & (1,14,6) & (9,3,13) & (5,1,9) & (2,15,12) & (9,8,12) \\
(12,13,8) & (11,10,2) & (14,8,4) & (10,1,8) & (13,12,3) & (3,9,11) & (7,4,10) \\
(13,6,15) & (2,4,13) & (14,7,5) & (9,2,10) & (15,14,13) & (0,10,4) & (6,11,12) \\
(12,5,15) & (15,6,8) & (5,3,12) & (7,11,13) & (13,11,1) & (6,13,7) & (4,15,7) \\
(1,10,11) & (8,2,5) & (13,5,10) & (13,14,0) & (12,9,7) & (12,6,10) & (5,14,11) \\
(12,1,4) & (8,9,1) & (10,9,15) & (10,5,7) & (10,3,0) & (14,15,9) & (10,6,14) \\
(15,0,5) & (1,7,15) & (8,0,14) & (11,9,6) & (13,9,4) & (0,13,2) & (2,9,14) \\
(9,5,0) & (4,6,9) & (4,12,0) & (1,5,13) & (15,3,10) & (15,2,11) & (11,15,4) \\
(3,15,1) & (0,8,15) & (12,14,2) & (11,3,14) & (11,5,8) & (7,14,3) & (4,8,11) \\
(0,12,11) & (11,0,7) & (7,1,12) & & & &
\end{array}
$$


Example 4.6. A DTS(18) that has no 18-good sequencing.

$$
\begin{array}{lllllll}
(1,2,3) & (4,3,2) & (3,4,5) & (6,5,4) & (5,6,2) & (7,2,6) & (2,7,8) \\
(3,8,7) & (8,3,6) & (2,1,0) & (6,0,1) & (0,6,3) & (6,11,16) & (9,0,17) \\
(11,15,3) & (10,12,8) & (3,15,11) & (15,14,13) & (17,15,2) & (11,7,4) & (5,16,13) \\
(10,2,17) & (17,3,14) & (0,9,10) & (12,13,2) & (13,1,8) & (2,4,10) & (7,10,3) \\
(12,7,0) & (6,9,7) & (12,15,10) & (1,17,16) & (8,2,13) & (2,9,16) & (2,12,5) \\
(7,11,13) & (12,17,6) & (13,11,9) & (16,9,8) & (6,17,13) & (16,11,2) & (13,12,3) \\
(10,13,5) & (13,0,16) & (4,0,11) & (3,0,13) & (11,5,1) & (17,1,7) & (8,1,11) \\
(15,7,1) & (16,7,14) & (14,5,0) & (9,15,4) & (14,11,6) & (14,16,3) & (4,1,15) \\
(0,14,7) & (10,4,9) & (10,14,1) & (9,5,3) & (9,13,14) & (1,13,10) & (17,10,11) \\
(14,8,10) & (7,15,9) & (17,0,5) & (11,10,0) & (5,10,7) & (5,11,12) & (0,8,12) \\
(2,11,14) & (16,17,4) & (15,16,12) & (8,0,4) & (5,14,15) & (4,8,16) & (16,1,5) \\
(3,10,16) & (6,15,8) & (6,12,14) & (4,17,12) & (13,4,7) & (3,12,1) & (16,6,10) \\
(0,2,15) & (9,1,6) & (10,15,6) & (1,4,14) & (14,12,4) & (7,12,16) & (13,15,17) \\
(3,17,9) & (16,15,0) & (7,5,17) & (14,9,2) & (8,14,17) & (8,15,5) & (4,13,6) \\
(11,17,8) & (5,8,9) & (1,9,12) & (12,9,11) & & &
\end{array}
$$

\section{Existence of $\operatorname{DTS}(v)$ without $v$-good sequenc- ings}

Let $K_{\mathrm{DTS}}^{*}=\{v \geq 3$ : there exists a DTS $(v)$ having no $v$-good sequencing $\}$. In this section, we prove that $v \in K_{\mathrm{DTS}}^{*}$ for all $v \equiv 0,1 \bmod 3, v \geq 7$.

We summarize results from Sections 3 and 4 in the following lemma.

Lemma 5.1. $3,4,6 \notin K_{\mathrm{DTS}}^{*}$ and $7,9,10,12,13,16,18 \in K_{\mathrm{DTS}}^{*}$.

Suppose $(Y, \mathcal{B})$ is a $\operatorname{DTS}(w)$ and $(X, \mathcal{A})$ is a $\operatorname{DTS}(v)$. We say that $(Y, \mathcal{B})$ is a subdesign of $(X, \mathcal{A})$ if $Y \subseteq X$ and $\mathcal{B} \subseteq \mathcal{A}$. The following lemma is obvious.

Lemma 5.2. Suppose that a DTS $(w)$ that does not have $w$-good sequencing is a subdesign of a DTS $(v)$. Then the DTS $(v)$ does not have v-good sequencing.

Theorem 5.3. Let $L=\{v \geq 3: v \equiv 0,1 \bmod 3\}$. Suppose $(X, \mathcal{B})$ is a $(v, L)-P B D$ and suppose there exists a block $B_{0} \in \mathcal{B}$ in the PBD such that $\left|B_{0}\right| \in K_{\mathrm{DTS}}^{*}$. Then $v \in K_{\mathrm{DTS}}^{*}$.

Proof. Replace every block $B$ of the PBD by a DTS $(|B|)$. For the block $B_{0}$, fill in a DTS $\left(\left|B_{0}\right|\right)$ that does not have a $\left|B_{0}\right|$-good squencing. The result follows from Lemma 5.2 . 
Corollary 5.4. Suppose $v \equiv 1,3 \bmod 6, v \geq 7$. Then $v \in K_{\mathrm{DTS}}^{*}$.

Proof. The values $v=7,9$ and 13 are handled in Lemma 5.1. From the Doyen-Wilson Theorem [7, there is an STS $(v)$ that contains an STS $(7)$ as a subdesign for all $v \geq 15, v \equiv 1,3 \bmod 6$. Replace the subdesign by a block of size 7 , obtaining a $(v,\{3,7\})$-PBD that contains a (unique) block of size 7. Because $7 \in K_{\mathrm{DTS}}^{*}$, the result follows from Theorem 5.3 .

Corollary 5.5. Suppose $v \equiv 0,4 \bmod 6, v \geq 10$. Then $v \in K_{\mathrm{DTS}}^{*}$.

Proof. The values $v=10,12,16$ and 18 are handled in Lemma 5.1, For $v \equiv 0,4 \bmod 6, v \geq 22$, write $v$ in the form $v=18 s+r$, where $r \in$ $\{4,6,10,12,16,18\}$ and $s \geq 1$. Because $v \geq 22$, we observe that

$$
\begin{aligned}
18 s+4 & =3(6 s+1)+1 \\
18 s+6 & =3(6 s+1)+3 \\
18 s+10 & =3(6 s+3)+1 \\
18 s+12 & =3(6 s+3)+3 \\
18 s+16 & =3(6 s+3)+7 \\
18 s+18 & =3(6 s+3)+9 .
\end{aligned}
$$

The first few equations in this series are $22=3 \times 7+1,24=3 \times 7+3$, $28=3 \times 9+1$ and $30=3 \times 9+3$. Thus, it is clear that we can express $v$ in the form $v=3 m+t$, where $m \equiv 1,3 \bmod 6, m \geq 7, m \geq t$ and and $t \in\{1,3,7,9\}$.

Now, take a transversal design $\operatorname{TD}(4, m)$ (see [4]) and delete $m-t$ points from one group. This gives rise to a $(v,\{3,4, m, t\})-\mathrm{PBD}$ that contains a block of size $m$. Corollary 5.4 proves that $m \in K_{\mathrm{DTS}}^{*}$, so the desired result follows from Theorem 5.3 .

Summarizing the results proven in Corollaries 5.4 and 5.5 and Lemma 5.1, we have the following.

Theorem 5.6. Suppose $v \equiv 0,1 \bmod 3, v \geq 3$. Then $v \notin K_{\mathrm{DTS}}^{*}$ if $v=3,4$ or 6 and $v \in K_{\mathrm{DTS}}^{*}$ if $v \geq 7$.

\section{Discussion and summary}

An interesting open question is if there is an efficient (i.e., polynomial-time) algorithm (perhaps using the ideas discussed in Section 4) to test if a given $\operatorname{DTS}(v)$ has a $v$-good sequencing. 
It would also be of interest to determine the proportion of DTS $(v)$ having a $v$-good sequencing among all DTS $(v)$ of a given order $v$. We ask if this ratio approaches 1 as $v$ increases.

Even for $v=9$, there are too many nonisomorphic designs to test them all. However, we did generate 10000 DTS(9) using our hill-climbing algorithm, and we determined that all but one of them has a 9-good sequencing (this exceptional design has an 8-good sequencing). For $v=10$, we again generated 10000 designs using our hill-climbing algorithm, and we found that they all have a 10 -good sequencing.

This suggests the following question: Does every DTS $(v)$ have either a $v$-good sequencing or a $(v-1)$-good sequencing?

\section{References}

[1] B. Alspach. Variations on the sequenceable theme. In "50 Years of Combinatorics, Graph Theory, and Computing", F. Chung, R. Graham, F. Hoffman, L. Hogben, R.C. Mullin, D.B. West, eds. CRC Press, 2020, to appear.

[2] B. Alspach, D.L. Kreher and A. Pastine. Sequencing partial Steiner triple systems. Preprint.

[3] M.J. Colbourn and C.J. Colbourn. Some small directed triple systems. Congr. Numer. 30 (1981), 247-255.

[4] C.J. Colbourn and J.H. Dinitz. Handbook of Combinatorial Designs, Second Edition, Chapman \& Hall/CRC, 2006.

[5] C.J. Colbourn and A. Rosa. Triple Systems, Oxford University Press, 1999.

[6] T.H. Cormen, C.E. Leiserson, R.L. Rivest, and C. Stein. Introduction to Algorithms, Third Edition. MIT Press, 2009.

[7] J. Doyen and R.M. Wilson. Embeddings of Steiner triple systems. Discrete Math. 5 (1973), 229-239.

[8] D.L. Kreher and D.R. Stinson. Nonsequenceable Steiner triple systems. Bull. Inst. Combin. Appl. 86 (2019), 64-68.

[9] D.L. Kreher and D.R. Stinson. Block-avoiding sequencings of points in Steiner triple systems. Australas. J. Combin. 74 (2019), 498-509. 
[10] D.L. Kreher, D.R. Stinson and S. Veitch. Good sequencings for small directed triple systems. Preprint.

[11] P.R.J. Östergård and O. Pottone. Classification of directed and hybrid triple systems. Bayreuth. Math. Schr. 74 (2005), 276-291.

[12] D.R. Stinson. Hill-climbing algorithms for the construction of combinatorial designs. In "Algorithms in Combinatorial Design Theory", NorthHolland, 1985, pp. 321-334 (Ann. Discrete Math., vol. 26).

[13] D.R. Stinson and S. Veitch. Block-avoiding point sequencings of arbitrary length in Steiner triple systems. Preprint. 